TITLE:

\title{
Negative photophoresis of smoke particles observed under microgravity
}

\section{$\operatorname{AUTHOR}(\mathrm{S}):$}

Watanabe, Shun N.; Iwaki, Takafumi; Kohira, Masahiro I.; Magome, Nobuyuki; Yoshikawa, Kenichi

\section{CITATION:}

Watanabe, Shun N....[et al]. Negative photophoresis of smoke particles observed under microgravity. Chemical Physics Letters 2011, 511(4-6): 447-451

\section{ISSUE DATE:}

2011-08

URL:

http://hdl.handle.net/2433/147050

\section{RIGHT:}

(C) 2011 Elsevier B.V.; This is not the published version. Please cite only the published version.; この論文は出版社版でありません。引用の際に は出版社版をご確認ご利用ください。 


\title{
Negative photophoresis of smoke particles observed under microgravity
}

\author{
Shun N. Watanabe ${ }^{\mathrm{a}, *}$, Takafumi Iwaki ${ }^{\mathrm{b}}$, Masahiro I. Kohira ${ }^{\mathrm{c}}$, Nobuyuki \\ Magome $^{\mathrm{d}}$, Kenichi Yoshikawa ${ }^{\mathrm{a}, *}$ \\ ${ }^{a}$ Department of Physics, Graduate School of Science, Kyoto University, \\ Kitashirakawa-oiwake-cho, Sakyo-ku, Kyoto 606-8502, Japan. Fax: +81-75-753-3779. \\ ${ }^{b}$ Fukui Institute for Fundamental Chemistry, Kyoto University, 34-4 Takano nishikai-cho, \\ Sakyo-ku, Kyoto 606-8103, Japan. \\ c Japan Space Forum, 2-2-1 Otemachi, Chiyoda-ku, Tokyo 100-0004, Japan \\ ${ }^{d}$ Institute for Integrated Cell-Material Sciences, Kyoto University, Yoshida \\ Ushinomiya-cho, Sakyo-ku, Kyoto 606-8501, Japan
}

\begin{abstract}
We observed the photophoresis of smoke particles in a small focal laser beam under micro-gravity, exerted in an airplane, to avoid air convectional flow due to buoyancy. We measured the motion of smoke particles and found that the particles exhibit negative photophoresis under the suppression of the convection. Based on the distribution of velocity of negative photophoresis, it is shown that the photophoretic velocity is proportional to the laser power applied for the particle. We discuss the mechanism in terms of the radiometric force.
\end{abstract}

\section{Introduction}

Transport phenomena have been attracting much interest not only from basic science but also from engineering and industry. Since the force applied to a transported object can originate from many physical processes, there are various forms of transport phenomena, such as electrophoresis, magnetophoresis, photophoresis and thermophoresis. In photophoresis, motion is induced by shining light on particles, which was first described by Ehrenhaft in 1917 [1]. Since photophoresis is observed for almost all types of particles $[1,2,3,4,5,6,7]$ unless they are completely transparent, it is expected that it can be applied to particles that do not have either an electric charge or a magnetic quality $[8,9,10,11]$.

Generally, photophoretic forces have two origins. One is called a scattering force (radiation pressure force) which is derived from the exchange of momentum between a photon and a scatterer [12]. The scattering force exerts pressure on a

\footnotetext{
* Corresponding author

Email addresses: watanabe@chem.scphys.kyoto-u.ac.jp (Shun N. Watanabe), yoshikaw@scphys.kyoto-u.ac.jp (Kenichi Yoshikawa)
} 
particle in the direction of the transferred momentum, which is in the direction of light.

The other is called a radiometric force (photo-thermal force) which arises from a surface-temperature-dependent reflection of ambient gas molecules [13, 14]. Under a kinetic boundary condition (Maxwell-type boundary condition), a difference in temperature occurs between gas molecules impinging on a particle surface and those departing from the particle surface. This temperature difference results in a deviation of the net pressure of the gas at the particle surface.

Therefore, a gradient in surface temperature leads to a gradient in the gas pressure at the particle surface, and thus, a photophoretic force arises in the direction from the hot side to the cold side of the particle. Photophoresis induced by radiometric force is very similar to thermophoresis. The difference is that in photophoresis the original temperature gradient exists on the particle surface, whereas in thermophoresis the ambient environment exhibits a temperature gradient over the size of the particle $[14,15,16]$.

Although, both the scattering force and the radiometric force work on a photo-induced motion of a particle, the radiometric force usually dominates photophoresis except for an ultra-high vacuum condition. [6] In the stratosphere and mesosphere where photophoresis is highlighted in conjunction with the behavior of aerosol particles, the effect of the scattering force is negligible, and photophoresis is mainly discussed in terms of the radiometric force [17].

In contrast to the scattering force, the radiometric force works either in the direction of light travel (positive photophoresis) or toward the light-source (negative photophoresis). This is because the heating power distribution caused by light absorption depends on the size and refraction index of the particle [18]. Since the scattering force always induces positive photophoresis, negative photophoresis is considered to be evidence of the radiometric force.

Photophoresis induced by the radiometric force is useful for many applications. The negative motion also means that the radiometric force is greater than scattering forces. Basically, the radiometric force increases under a higher ambient air pressure. However, in an actual experiment, we have to take into account another factor that disturbs photophoretic motion. Since a container is more or less heated by light, natural convection due to buoyancy occurs in the experimental system when the experiment is performed in a usual laboratory. In experiments under natural atmospheric pressure, it is difficult to eliminate the possibility that natural convection influences the direction and speed of photophoresis.

In this Letter, we performed the experiment of the photophoresis of smoke particles under micro-gravity by using an airplane. In one flight, we can measure both the case of $1.0 \mathrm{~g}$ and $0.0 \mathrm{~g}$. We observed convection of ambient air for the result at $1.0 \mathrm{~g}$ and did not observe convection at $0.0 \mathrm{~g}$. From the result at 0.0 $g$, we obtained the relationship between photophoretic force and light intensity. 


\section{Materials and methods}

Figure 1 shows the experimental setup. We generated smoke particles by burning a sandalwood incense stick (product name is "Hanafuzei" from Kameyama, Japan). Main composite of a smoke particle is estimated as water and some organic acid because it is generated from burning organic materials. The diameter of the smoke particles was measured by dynamic light scattering with a SGS-6000 (Otsuka Electronics, Japan) 2 min later after the generation. The measured mean diameter was $0.78 \pm 0.20 \mu \mathrm{m}$. Just before the observation under microgravity by $2 \mathrm{~min}$, the smoke particles were generated in a syringe and injected into a $10 \mathrm{~mm}$ cubic optically transparent cell. The laser-transmitting sides of the cell were made of glass plates and the other sides were made of acrylic plates.

The laser source was a frequency-doubled (532 nm) diode-pumped, solidstate $\mathrm{Nd}: \mathrm{YVO}_{4}$ laser (Millennia, Spectra-Physics), which delivers a continuous $\mathrm{TEM}_{00}$ beam. The output power is $5.0 \mathrm{~W}$. In the present microgravity experiment, the measurement time was limited to about $10 \mathrm{~s}$. To take various photophoretic intensities at once, we used a focal beam system. To make the focal beam system, we used a lens with a focal length of $10 \mathrm{~mm}$. The final beam convergence angle was 7.0 degrees.

We measured the radiation power with a power meter (PM-345, Neoarc Japan) at each point. We used a microscope CCD camera (VH-Z75, Keyence, Japan) to observe the particles by light scattering. The video images were recorded on a digital video recorder (DSR-11, SONY, Japan).

All of the experimental systems were installed in an airplane (Mitsubishi MU-300, operated by Diamond Air Service Co., Japan). By taking a parabolic flight-path with the airplane, we achieved a micro-gravity condition. The microgravity condition was maintained for about $10 \mathrm{~s}$ and the residual gravity was maintained at less than $0.01 \mathrm{~g}$ ( $\mathrm{g}$ is the standard acceleration due to on Earth, $9.8 \mathrm{~m} \mathrm{~s}^{-2}$ ). A microgravity environment can be used to avoid thermal convection of an ambient gas caused by heating of the experimental system [19, 20, 21]. The air conditions were regulated at $293 \pm 3 \mathrm{~K}$ and 0.9 atm.

In our experiment, a nearly Gaussian beam $\mathrm{TEM}_{00}$ mode was generated. While the optical fiber may disturb the beam profiles to a certain degree, the spatial profile of the light intensity of the mixed mode focal beam can be described by the following equation $[22,23]$ :

$$
\begin{aligned}
I(h, z) & =\frac{P_{\text {focal }}}{\pi \sigma^{2}(z)} \exp \left(-\frac{h^{2}}{\sigma^{2}(z)}\right) \\
\sigma(z) & =\sqrt{W_{0}^{2}+\left\{\tan \left(\theta_{\text {focal }}\right) z\right\}^{2}},
\end{aligned}
$$

where $h$ is the axis normal to the optical axis, $z$ is taken along the optical axis with $z=0$ as the focal point, $P_{\text {focal }}$ is the total laser power of the focal beam, $W_{0}$ is a constant of the minimum focal spot, and $\theta_{\text {focal }}$ is the convergence angle. We measured $P_{\text {focal }}=0.26 \mathrm{~W}$ when the output power of the laser head was set as $5.0 \mathrm{~W}$. 


\section{Results}

Figure 2 shows CCD camera images near the focal point. Figure 2(a) shows snapshots of the focal beam with a time resolution of $1 / 30 \mathrm{~s}$. The bright spots, which indicate smoke particles, are seen to concentrate on the center line. In fact, smoke particles are present throughout the entire experimental cell, but only particles that are irradiated by the laser beams are seen in the figure by scattering light. The actual beam power profiles can be estimated from the scattering intensity.

Figure 2(b) shows time-accumulated images of the scattering light intensity. By fitting the intensity profiles in Figure 2(b) with Eqs. (1) and (2), we evaluated the experimental parameters characterizing that characterized the focal beams; $W_{0}=0.10 \mathrm{~mm}$, and $\theta_{\text {focal }}=7.0$ degrees.

Figures 3(a) (at $1.0 \mathrm{~g}$ ) and (b) (at $0.0 \mathrm{~g})$ is spatiotemporal plots of the light axis of the focal beam and the spatial distributions of photophoretic velocities. In each figure, the irradiated light comes from the left. Most of the particles move toward the light source. The effect of radiometric force is very sensitive to the size of the particle. In Figure 3, it is found that small fraction of particles exhibit no motion or positive photophoresis, being attributed to other kinds of "dust" particles or object with large size difference from usual smoke particles. Here we will pay our attention on the large fraction of the particles, showing negative photophoresis, for which the radiometric force is clearly dominant. At $1.0 \mathrm{~g}$, owing to the existence of air convection, the distribution was deviated from the symmetry with respect to the focal point, $z=0$. On the other hand, in the system at $0.0 \mathrm{~g}$, we successfully measured the photophoretic velocity distribution by diminishing the effect of air convection.

\section{Discussion}

Since the scattering force always induces positive photophoresis, the presence of negative photophoresis is regarded as the actual evidence that the dominant force is the radiometric force [10]. Orr and Keng [24], Tong [16] and Lin [6] associated their experimental observations of negative photophoresis with the radiometric force, based on the fact that photophoretic force decreases with a decrease in atmospheric pressure.

In our experiment, the pressure was not low, being on the order of the atmospheric pressure on earth. As seen in Figure 3, the photophoretic motion was disturbed by thermal convection in air under $1.0 \mathrm{~g}$. On the other hand, effect due to convective flow was not seen under $0.0 \mathrm{~g}$.

Figure 4(a) shows the averages and standard deviations of the velocities based on the lower panels in Figure 3(b). The solid curve shows the estimated light intensity at each point calculated from Eq. (1). The velocities show a symmetric profile, which suggests that inertia does not have much of an effect, and that the velocity data are very close to their respective stationary values. Since the light intensity is different at each point, the position-dependent velocity distribution gives a relation between the velocity and light intensity. Figure 
4(b) is a log-log plot of the average velocity of smoke particles versus light intensity. The relationship between the negative velocity $-u$ and the light intensity $I$ shows a scaling relation over a wide range of light intensity.

Based on the results shown in Figure 4, the relationship between photophoretic velocity and light intensity is deduced to be $u_{\mathrm{ph}} / I=-4.0 \times 10^{-10} \mathrm{~m}^{3} / \mathrm{J}$. As the Reynolds number of this system is very low $\left(\mathrm{Re} \simeq 10^{-3}\right)$, the particle inertia is negligible for a video frame of $1 / 30 \mathrm{~s}$. Thus, the photophoretic velocity only follows the photophoretic force. From Stokes' law, we can describe the photophoretic force as

$$
F_{\mathrm{ph}}=6 \pi \nu R_{0} u_{\mathrm{ph}}
$$

where $\nu$ is the viscosity of air and $R_{0}$ is the radius of the particle. If we use the values $\nu=1.82 \times 10^{-5} \mathrm{~Pa} \cdot \mathrm{s}[25]$ and $R_{0}=3.9 \times 10^{-7} \mathrm{~m}$, we obtain $F_{\mathrm{ph}} / I=-5.4 \times 10^{-20} \mathrm{~m} \cdot \mathrm{s}$.

Under the framework of Chernyak and Beresnev [26], the photophoretic force is calculated as

$$
\begin{aligned}
F_{\mathrm{ph}} & =-\frac{\pi}{3} R_{0}^{2} I J_{1}\left(\frac{\pi m}{2 k T_{\mathrm{g}}}\right) \frac{\alpha_{\mathrm{E}} \psi_{1}}{\alpha_{\mathrm{E}}+15 \Lambda \mathrm{Kn}\left(1-\alpha_{\mathrm{E}}\right) / 4+\alpha_{\mathrm{E}} \Lambda \psi_{2}}, \\
J_{1} & =-3 \operatorname{Re}(n) \operatorname{Im}(n) \frac{2 \pi R_{0}}{\lambda} \int_{0}^{\pi} \sin \theta \cos \theta d \theta \int_{0}^{1} \frac{|\mathbf{E}(\bar{x}, \theta)|^{2}}{\left|\mathbf{E}_{0}\right|^{2}} \bar{x}^{3} d \bar{x} \\
\Lambda & =\frac{\kappa_{\mathrm{p}}}{\kappa_{\mathrm{g}}} \\
\psi_{1} & =\frac{\mathrm{Kn}}{\mathrm{Kn}+\frac{5 \pi}{18}}\left(1+\frac{2 \pi^{1 / 2} \mathrm{Kn}}{5 \mathrm{Kn}^{2}+\pi^{1 / 2} \mathrm{Kn}+\pi / 4}\right), \\
\psi_{2} & =\left(\frac{1}{2}+\frac{15}{4} \mathrm{Kn}\right)\left(1-\frac{1.21 \pi^{1 / 2} \mathrm{Kn}}{100 \mathrm{Kn}^{2}+\pi / 4}\right) \\
\mathrm{Kn} & =\frac{l}{R_{0}}
\end{aligned}
$$

where $I$ is an irradiated light intensity, $J_{1}$ is an asymmetry factor, $\mathbf{E}$ is an electric field, $\left|\mathbf{E}_{0}\right|$ is the incident electric field strength, $\bar{x}=\frac{2 r}{a}$ is a radial distance normalized by diameter of the particle, $m$ is the mass of the ambient gas molecule, $T_{\mathrm{g}}$ is the temperature of the ambient gas, $k$ is the Bolzmann constant, $\alpha_{\mathrm{E}}$ is a thermal accommodation coefficient for energy on the surface of the particle, $\kappa_{\mathrm{p}}$ is a thermal conductivity of the particle, $\kappa_{\mathrm{g}}$ is a thermal conductivity of the gas, $\mathrm{Kn}$ is the Knudsen number, and $l$ is the mean free path of the gas. According to our experimental condition, we adopt $J_{1}=2.5 \times 10^{-3}$, which is calculated by a refractive index of cigarette smoke $n=1.45-0.0013 i[27]$ into equations of Ref. [28], $m=2.3 \times 10^{-26} \mathrm{~kg}$ for nitrogen, $k=1.3 \times 10^{-23} \mathrm{~J} / \mathrm{K}, T_{\mathrm{g}}=$ $293 \mathrm{~K}, \alpha_{\mathrm{E}}=1, \kappa_{\mathrm{p}}=0.56 \mathrm{~W} /(\mathrm{m} \cdot \mathrm{K})$ for water [25], $\kappa_{\mathrm{g}}=0.024 \mathrm{~W} /(\mathrm{m} \cdot \mathrm{K})$ for air [25], and $l=7.27 \times 10^{-8} \mathrm{~m}$ for air at 0.9 atm [29]. Thus, we obtain $F_{\mathrm{ph}} / I=-3.8 \times 10^{-23} \mathrm{~m} \cdot \mathrm{s}$. This force is much smaller than our experimental results. Because a slip condition is applied on the surface of a photophoretic particle, the drag force does not necessarily obey the Stokes' law. Moreover, 
a temperature distribution would change between the conditions at rest and in a steady motion. Using the theory [30] based on the slightly rarefied gas dynamics, we obtain a relationship between the photophoretic velocity and the light intensity as $u_{p h} / I=-1.2 \times 10^{-10} \mathrm{~m}^{3} / \mathrm{J}$. Taking into the rough estimation of the parameters, this value is compatible with the experimental result.

There is a chance that an internal motion of a particle affects the temperature gradient.Here, we consider the effect of rotational Brownian motion of a particle. The average angular velocity of the particle for a Brownian particle is estimated as $\sqrt{18 k T /\left(\pi \rho a^{3}\right)} \simeq 6.8 \times 10^{-3} \mathrm{rad} / \mathrm{s}$. On the other hand, the thermal diffusivity of water normalized by the diameter of the particle is $2.3 \times 10^{5} \mathrm{~s}^{-1}$. The thermal diffusivity of air normalized by the diameter of the particle is $3.6 \times 10^{7} \mathrm{~s}^{-1}$. Thermal diffusion is much faster than the rotational Brownian motion, and thus, the effect of Brownian motion would be negligible. Recently, it was predicted [30] based on a theoretical argument that the photophoresis of a fluid particle shows a complex behavior because of a convectional flow in the particle. The convectional flow is expected to dominate the temperature distribution when the diameter is larger than submicrometer scale. Further experiments in microgravity are expected to contribute to the discovery and confirmation of such new mechanics.

Scattering force for the smoke particle is roughly estimated as

$$
\frac{F_{\mathrm{sc}}}{I} \simeq \pi R_{0}^{2} \frac{1-\exp \left(2 \pi R_{0} \operatorname{Re}(n) \operatorname{Im}(n) / \lambda \mu c\right)}{c}=7.4 \times 10^{-26} \mathrm{~m} \cdot \mathrm{s},
$$

where $\mu=4 \pi \times 10^{7}$ is magnetic permeability and $c=3.0 \times 10^{8} \mathrm{~m} \mathrm{~s}^{-1}$ is light speed. By increasing ambient gas pressure, scattering force takes a constant value while radiometric force highly depends on the pressure. In the present experimental condition, the scattering force is actually negligible. On earth at $1 \mathrm{~g}$ environment, air convection is also increased and disturbs the manipulation. In the present study, we have made it clear that this disturbance does not occur in a microgravity environment. A better understanding of negative photophoresis should contribute to manipulations in microgravity, such as in a chemical laboratory in a Space Station.

\section{Concluding remarks}

We observed the negative photophoresis of smoke particles from a burned stick of incense in air at $0.9 \mathrm{~atm}$. The results at $1.0 \mathrm{~g}$ showed convective flow and this flow affected to the velocities of particles. This caused the difficulty to map the spatial distribution of velocity in the relationship between photophoretic velocity and irradiated light intensity. On the contrary, the results at $0.0 \mathrm{~g}$ did not exhibit the disturbance by convective flow and we could map the spatial distribution of photophoretic velocities into a relationship between photophoretic velocity and irradiated light intensity. The linear relationship thus experimentally obtained is consistent with the theoretical expectation. The results are consistent with the theoretical estimate. 


\section{Acknowledgments}

The authors thank Mr. N. Matsuda for his helpful discussion regarding the experiments, Dr. Y. Sumino for helping with the experiments, Dr. A. Isomura for his helpful discussion on image processing, and Ms. Hayata and Messes. Y. Fujii, M. Kawakatsu and S. Takahashi for their technical assistance. This research was supported by "Ground-based Research Announcement for Space Utilization" promoted by the Japan Space Forum. This research was also supported by a grant from the Ministry of Education, Science, Sports and Culture of Japan (No. 22654046).

\section{References}

[1] F. Ehrenhaft, Phys. Z. 18 (1917) 352.

[2] S. G. Warren and W. J. Wiscombe, J. Atmos. Sci. 37 (1980) 2734.

[3] H. Rosen, T. Novakov, and B. A. Bodhaine, Atmos. Environ. 15 (1981) 1371.

[4] V. V. Karasev, N. A. Ivanova, A. R. Sadykova, N. Kukhareva, A. M. Baklanov, A. A. Onischuk, F. D. Kovalev, and S. A. Beresnev, J. Aerosol Sci. 35 (2004) 363.

[5] V. G. Chermyak, and S. A. Beresnev, J. Aerosol Sci. 24 (1993) 857.

[6] H. B. Lin, Opt. Lett. 10 (1985) 68.

[7] E. R. Buckle and Y. M. Bushnell-Watson, J. Coll. Interface Sci. 105 (1985) 529.

[8] H. Watarai, H. Monjushiro, S. Tsukahara, M. Suwa, and Y. Iiguni, Anal. Sci. 20 (2004) 423.

[9] T. Imasaka, Y. Kawabata, T. Kaneta, and Y. Ishidzu, Anal. Chem. 67 (1995) 1763.

[10] T. X. Phuoc, Opt. Commun. 245 (2005) 27.

[11] T. W. Ng, A. Neild, and P. Heeraman, Opt. Lett. 33 (2008) 584.

[12] A. Ashikin, Phys. Rev. Lett. 24 (1970) 156.

[13] A. Rubinowicz, Ann. Phys. 62, (1920) 691.

[14] E. H. Kennard, Kinetic Theory of Gases, McGraw-Hill Company, Columbus, 1938, p. 327.

[15] D. W. Mackowski, Int. J. Heat Mass Transfer 32 (1989) 843.

[16] N. T. Tong, J. Coll. Interface Sci. 51 (1975) 143. 
[17] G. Wurm and O. Krauss, Atmos. Environ. 42 (2008) 2682.

[18] M. Sitarski, and M. Kerker, J. Atmos. Sci. 41 (1984) 2250.

[19] J. Steinbach, J. Blum, and M. Krause, Eur. Phys. J. E 15 (2004) 287.

[20] M. Tamagawa, H. Monjushiro, and H. Watarai, Colloids Surf. A 220 (2003) 279 .

[21] H. Monjushiro, K. Takeuchi, and H. Watarai, Chem. Lett. 31 (2002) 788.

[22] H. Kogelnik, and T. Li, Appl. Opt. 5 (1966) 1550.

[23] T. F. Johnston, LASER FOCUS World 26 (1990) 173.

[24] C. Orr, and E. Y. H. Keng, J. Atmos. Sci. 21 (1964) 475.

[25] G. W. C. Kaye and T. H. Laby, Tables of physical and chemical constants and some mathematical functions, Longman, London, 1986.

[26] V. Chernyak, and S. Beresnev, J. Aerosol Sci., 24 (1993) 857.

[27] M. Kerker, M. J. Sculley, W. A. Farone and A. J. Kassman, Appl. Opt., 17 (1978) 3030.

[28] P. W. Dusel, M. Kerker and D. D. Cooke, J. Opt. Soc. Am., 69 (1979) 55.

[29] S. G. Jennings, J. Aerosol Sci. 19 (1988) 159.

[30] T. Iwaki, Phys. Rev. E 81 (2010) 066315. 


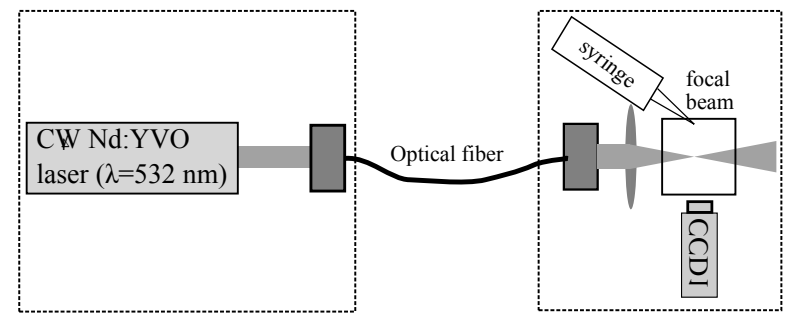

Figure 1: Schematic illustration of the experimental setup. The experiments were performed in an airplane.

(a)

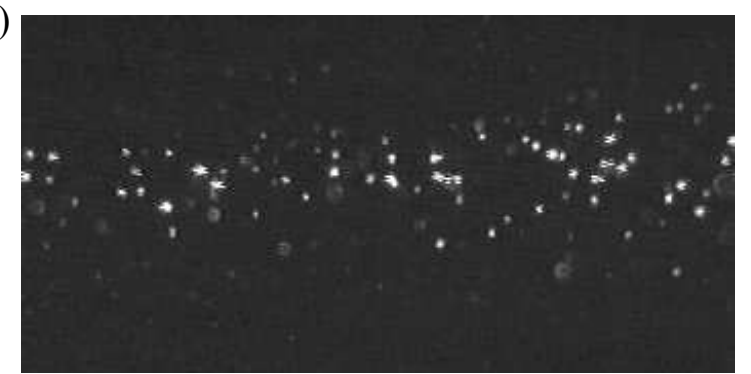

(b)

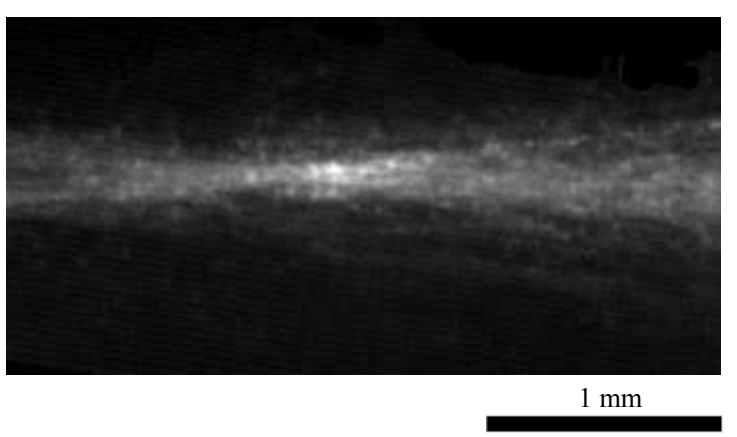

Figure 2: CCD camera images of smoke irradiated by a laser beam from the left side. (a) Snapshot with a time resolution of $1 / 30 \mathrm{~s}$ and (b) time-accumulation image for a period of 10 

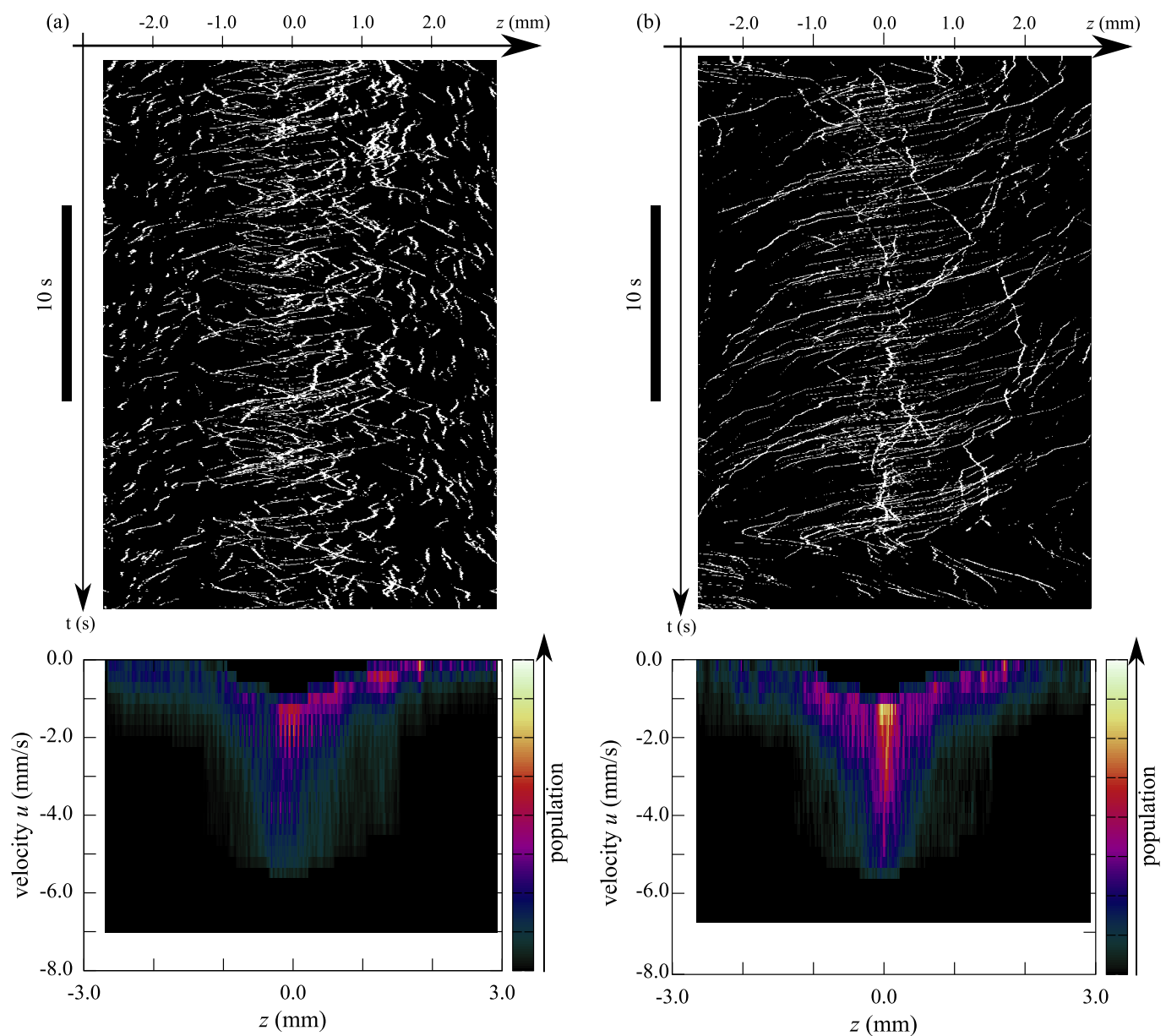

Figure 3: Spatio-temporal plots along the light axis (above), and the photophoretic velocity distributions of smoke particles (below). (a) is the experiment at $1.0 \mathrm{~g}$ in level flight and (b) is at $0.0 \mathrm{~g}$ on a parabolic path. As for the velocity maps in the lower panels, we eliminated a small number of particles exhibiting no apparent motion or positive photophoresis. 
(a)
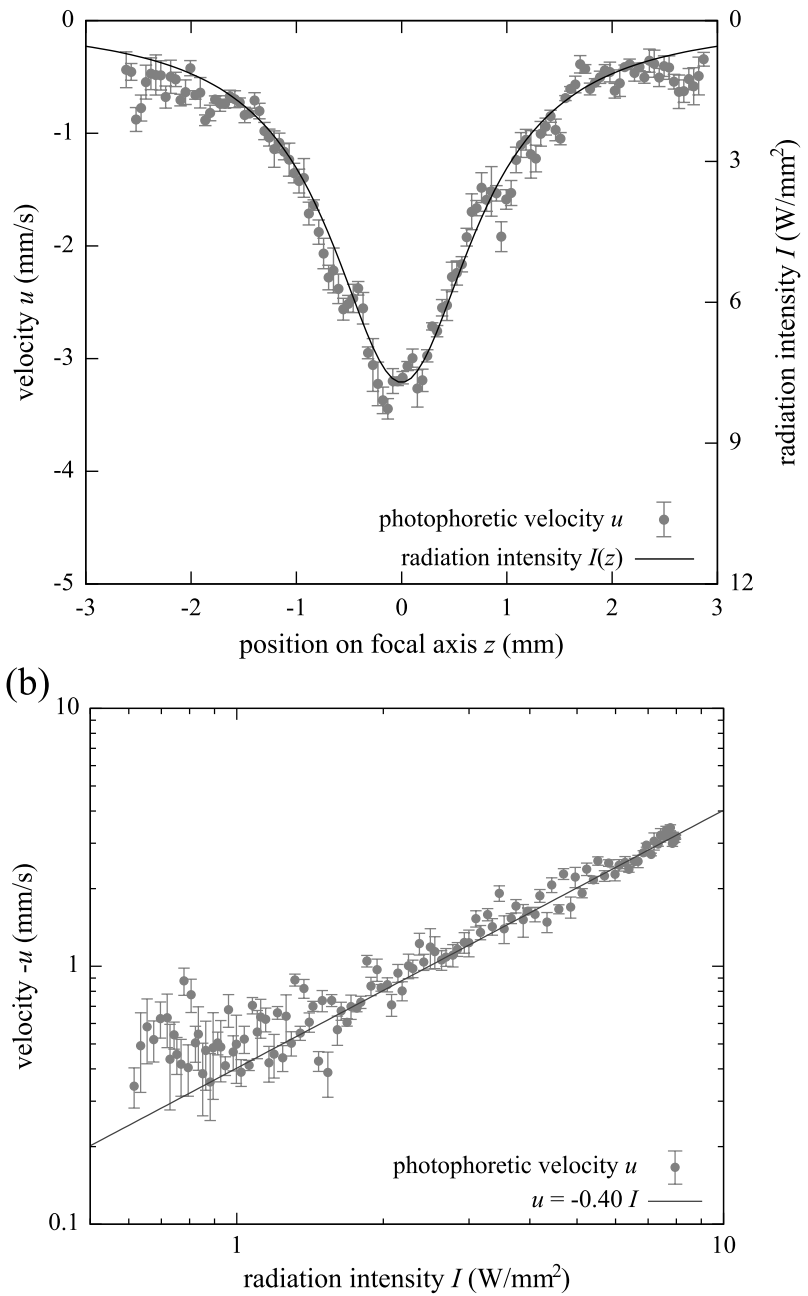

Figure 4: (a) Spatial distribution of the photophoretic velocities at $0.0 \mathrm{~g}$ along the optical axis together with the standard deviations as vertical bars. The light intensity distribution, $I(z)$, is also shown as a solid curve. (b) Log-log plot of negative photophoretic velocities $-u$ (the value of $u$ itself is negative) versus light intensity $I$. The solid line is the fitting line to the velocities. 